\title{
Photonic generation of broadband RFphase shifter
}

This paper was downloaded from TechRxiv (https://www.techrxiv.org).

\section{LICENSE}

CC BY 4.0

SUBMISSION DATE / POSTED DATE

$18-07-2020$ / 26-07-2020

CITATION

Hasan, Mehedi (2020): Photonic generation of broadband RFphase shifter. TechRxiv. Preprint. https://doi.org/10.36227/techrxiv.12671150.v1

$\mathrm{DOI}$

10.36227/techrxiv.12671150.v1 


\title{
Photonic generation of broadband RF phase shifter
}

\author{
Mehedi Hasan \\ Centre for Research in Photonics \\ University of Ottawa \\ Ottawa, Canada \\ mhasa067@uottawa.ca
}

\author{
Trevor Hall \\ Centre for Research in Photonics \\ University of Ottawa \\ Ottawa, Canada \\ Trevor.hall@uottawa.ca
}

\begin{abstract}
The circuit architecture of a compact and broadband RF phase shifter is presented along with simulation verification. The complex transmission of the phase shifter follows a trajectory on a unit circle that may rotate around the origin an arbitrary number of times in either direction.
\end{abstract}

Keywords—photonic phase shifter, phased array, SSB-SC, DP-MZM

\section{INTRODUCTION}

A precise and broadband radio frequency $(\mathrm{RF})$ phase shifter is a growing need for phased array antenna system to support high speed wireless communication and radar applications. The photonic generation of microwave phase shifts is receiving enormous attention due to its simplicity. light weight, low cost, small size and broadband operation with minimal phase errors. The underlying principle is based on frequency shifting an optical carrier via single side band -suppressed carrier (SSB-SC) modulation using RF carrier of interest followed by the re-insertion of a copy of the original optical carrier that is shifted in phase by a variety of means such as optical phase modulator [1], p-n junction [2], z-cut lithium niobite crystal $\left(\mathrm{LiNbO}_{3}\right)$ [3] and using precise setting of polarization controller [4]. All these methods require either an optical deinterleaver filter [2], precise polarization control and polarizer [3-4] or suffers from lack of phase shift linearity and range. We proposed a circuit architecture based on a parallel pair of dual-parallel Mach-Zehnder modulators (DP-MZM) that requires no additional control or filter for proper operation. Furthermore, the linearity of the phase shifter remains valid for a wide range of frequencies. The proposed phase shifter can generate any phase without bound. The complex transmission of the phase shifter follows a trajectory in the complex plane that may encircle the origin an arbitrary number of times in either direction. The practical implementation of the proposed design can be demonstrated using a commercially available dual polarization quadrature phase shift keying (DP-QPSK) modulator. 


\section{PRINCIPAL OF OPERATION}

The circuit diagram of the proposed braodband phase shifter is given in fig. 1 . The circuit consists of two DP-MZM. The upper DP-MZM generates the suppressed carrier-single side band modulation of the applied RF drive signal. To obtain this function, the bias of the two parallel child MZM is set to their minmum tranmission points (MITP), while the mother MZM is set to its quadrature transmission point (QTP). Then the in-phase (I) and quadrature-phase $(\mathrm{Q})$ of the $\mathrm{RF}$ drive signal is applied to the upper and lower child MZM respectively [5]. SSB-SC modulation can also be regarded as the shifting of the optical carrier frequency by an amount equal to the RF frequency. The bias of the lower DP-MZM is set similarly. A baseband or low frequency in-phase and quadrature-phase signal is applied to the two child MZM to shift the phase of the optical carrier. When the output of the two DP-MZM imping on a photodiode, the phase shifted version of the RF drive signal is recovered.
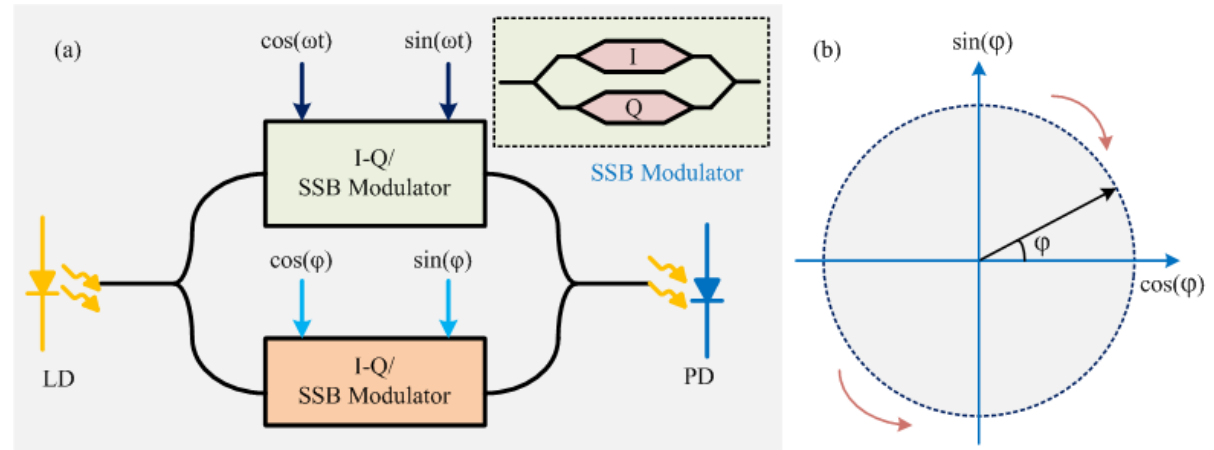

Fig. 1. (a) Schematic diagram of the proposed broadband phase shifter; (b) phase shift trajectory (either clock wise or anticlock wise); LD, Laser diode; SSB, Single side band; PD, Photodiode.

In the small modulation index $\left(\mathrm{m}_{1}\right)$ approximation, the transmission of the upper DP-MZM is $(1 / 4) \mathrm{m}_{1}\left(\exp \left(\omega_{\mathrm{c}}+\omega_{\mathrm{rf}}\right) \mathrm{t}\right)$, where, $\omega_{\mathrm{c}}$ and $\omega_{\mathrm{rf}}$ is the optical and RF carrier frequency. The output transmission of the lower DP-MZM can be written: $(1 / 4) \mathrm{m}_{2}\left(\exp \left(\omega_{\mathrm{c}}+\varphi\right) \mathrm{t}\right)$, where $\varphi$ is the applied phase shift to the optical carrier through I-Q modulation at the lower DP-MZM. Finally, the beat signal of interest at the output of the photodiode is $\alpha \exp \left(\left(\omega_{m}-\varphi\right) t\right)$. 


\section{Simulation RESUlt}

Circuit simulation using VPIphotonics is performed to verify the theoretical prediction. A Distributed Feedback (DFB) laser having wavelength of $1550 \mathrm{~nm}$ and an output power of $10 \mathrm{dBm}$ is used as the optical source. The insertion loss and extinction ratio of the each MZM is considered to be $4 \mathrm{~dB}$ and $30 \mathrm{~dB}$ respectively. The half-wave voltage of each MZM is considered to be $5 \mathrm{~V}$. The in-phase and quadrature phase of the RF drive signal with peak amplitude of $1.2 \mathrm{~V}$, having a frequency of $10 \mathrm{GHz}$ is applied to the upper DP-MZM. This results in the shift of the optical carrier by an amount of $10 \mathrm{GHz}$. Although suppressed-carrier modulation is desired, the suppression is not complete due to the finite extinction of the MZM. For the purpose of demonstrating the operating principle by simulation, a very low frequency cosine- and sine- waveform are applied to the inphase and quadrature-phase inputs to the lower DP-MZM to generate a slow linear ramp of the optical carrier phase. Once both the output of the upper and lower DP-MZM are combined at the photodiode, the desired phase shifted RF carrier can be observed. Figure 2 shows the phase shift at

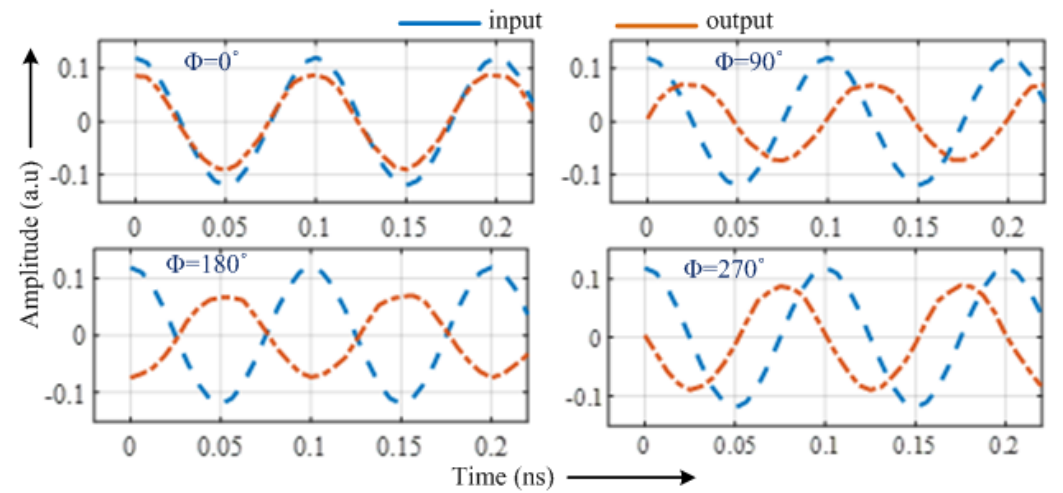

Fig. 2. Simulated phase relationship between the input and output RF signal.

different static phase $(\varphi)$ of the low frequency drive signal predicted by the simulation. The output phase shift exactly matches the applied phase shift and without bound. It can rotate about the origin in either direction (clockwise and anti-clockwise) an arbitrary number of times. time. The power variation of the recovered signal at different phase is obtained $\sim 1.8 \mathrm{~dB}$ maximum. This variation is the result of the nonlinear transmission function of the DP-MZM and may be corrected by predistortion of the in-phase and quadrature phase controls or alternative methods [NTT ref] of the lower DP-MZM. The proposed circuit can be used up to the bandwidth of the DP-MZM.

\section{CONCLUSION}

In summary, a novel photonic technique for obtaining the phase shift of a RF signal is reported with simulation verification. The proposed circuit can function for a wide range of frequencies and it can generate a phase without bound. The circuit may be implemented in practice using a commercially available DP-QPSK modulator.

\section{ACKNOWLEDGMENT}

Mehedi Hasan acknowledges the Natural Sciences and Engineering Research Council of Canada (NSERC) for their support through the Vanier Canada Graduate Scholarship program. Trevor J. Hall is also grateful to the University of Ottawa for their support of a University Research Chair. 


\section{REFERENCES}

[1] J. Han, B. Seo, S. Ku. Kim, H. Zhang and H. R. Fetterman, "Single-chip integrated electro-optic polymer photonic RF phase shifter array,” J. Lightwave Technol., vol. 21, pp. 3257-3261, 2003.

[2] C. Porzi, G. Serafino, M. Sans, F. Falconi, V. Sorianello, S. Pinna, J. E. Mitchell, M. Romagnoli, A. Bogoni, and P. Ghelfi , "Photonic Integrated Microwave Phase Shifter up to the mm-Wave Band With Fast Response Time in Silicon-on-Insulator Technology," J. Lightwave Technol., vol. 36, pp. 44944500, 2018

[3] T. Niu, X. Wang, E. H. W. Chan, X. Feng and B. O. Guan, "Dual-Polarization Dual-Parallel MZM and Optical Phase Shifter Based Microwave Photonic Phase Controller," IEEE Photonics Journal, vol. 8, pp. 1-14, 2016.

[4] S. Zhu, M. Li, X. Wang, N. Hua Zhu, and W. Li, " $1 \times$ N hybrid radio frequency photonic splitter based on a dual-polarization dual-parallel Mach Zehnder modulator," Optics Communications, vol. 431, pp. 10-13, 2019.

[5] R. Maldonado-Basilio et al., "Electro-optic up-conversion mixer amenable to photonic integration," Journal of Modern Optics, vol. 62, pp. 1405-1411, 2015.

[6] H. Yamazaki, H. Takahashi, T. Goh, Y. Hashizume, T. Yamada, S. Mino, H. Kawakami, and Y. Miyamoto, "Optical Modulator With a Near-Linear Field Response," J. Lightwave Technol., vol. 34, pp. 3796-3802 (2016) 